\title{
RF Cavity Design for High Current Operation of the Cornell Electron Storage Ring*
}

\author{
S. Greenwald, Z. Greenwald, D. Hartill, D. Morse, J. Kirchgessner, D. Rice
}

Laboratory of Nuclear Studies, Cornell University, Ithaca NY 14853

\begin{abstract}
Redesign of the $500 \mathrm{MHz}$ RF cavities used in Cornell Electron Storage Ring (CESR) has been carried out to improve their reliability and to optimize them for $600 \mathrm{~mA}$ total current. The factor of four higher beam current compared to the present operation levels requires redesign of the quarter wave input coupling transformer, the 50 ohm coaxial ceramic high power window and improvement of the power handling capability of the higher order mode load on each cell to $20 \mathrm{~kW}$. To lower the higher order mode impedance of the cavities and to simplify their mechanical construction, the number of cells in each cavity has been decreased from 14 to 5 cells and the number of cavities has been increased from 2 to 4 cavities. Design details, modeling and computer simulation of the expected performance are presented.
\end{abstract}

\section{Introduction}

The Laboratory of Nuclear Studies at Cornell University is upgrading the luminosity of the CESR Storage Ring from peak luminosity of $1 \times 10^{\star \star} 32$ $\left[1 / \mathrm{cm}^{* *} 2 \mathrm{sec}\right]$. to $5 \times 10^{* *} 32$ [1/ $\left.\mathrm{cm}^{* *} 2 \mathrm{sec}.\right]$. Besides changes in the configuration of the magnetic guide field for the stored electrons and positrons, the upgrade plan calls for a factor of four increase in the total stored current of electrons and positrons from the present $150 \mathrm{~mA}$ to $600 \mathrm{~mA}$. To provide for the increased power lost to synchrotron radiation and to higher order RF modes in the RF cavities, the vacuum chamber and the electrostatic seperators of the storage ring by this increased current, at least two new RF cavities must be added to the present two RF cavities in CESR. The final goal is to replace the present two RF cavities with two additional new ones. The present RF cavities [1], [2] have been in service in CESR for the past 10 years.

\section{Design Guide Lines}

Reliability: In order to improve the reliability and vacuum performance of the cavities, the welding techniques for joining various metal parts have been changed which required a complete new mechanical design. The only welds permitted in the new cavities are:

a. Copper to Copper -..............-Electron beam.

b. Copper to Stainless Steel-.....--Vacuum Braze. c. Stainless steel to Stainless Steel-TIG.

Higher Order Modes: With a total current of $600 \mathrm{~mA}, 15$ to $20 \mathrm{~kW}$ will be stored in the higher order modes in each cell. Therefore to be able to dissipate this power a $30 \mathrm{~kW}$ water cooled load is attached to each cell with the coupling to the high order mode coupler made by a 10 degree coaxial tapered elbow in which the center conductor is water cooled. (In the present cavities we use a 10 kW water cooled load and a 45 degree coaxial elbow).

Maintenance: In order to simplify the maintenance and to shorten the repair time, the cooling water tank, in which the cavity is submerged, is built in such a way that each part of the cavity can be reached without removing the cavity from the storage ring by removing the appropriate side wall of the tank. The $30 \mathrm{~kW}$ loads are placed outside the water tank which enables us to monitor each load separately. In case of failure the replacement of a defective load will take about one hour. The same procedure in the present cavities takes a few days.

\section{Cavity Description}

Each cavity, a drawing of which can be seen in Figure 1. consists of five major subassemblies:

a. A $50 \mathrm{ohm}$ coaxial ceramic high power vacuum window. A drawing of which can be seen in Figure 2.

b. A quarter wave transformer to match the 50 ohm coaxial window to the 30 ohm feed line.

c. Five resonant cell RF cavities.

d. 30 ohm coaxial feed line.

e. A tuner which provides the overall tuning of the five cells, the coaxial feed line and the window.

*Work supported by the National Science Foundation 


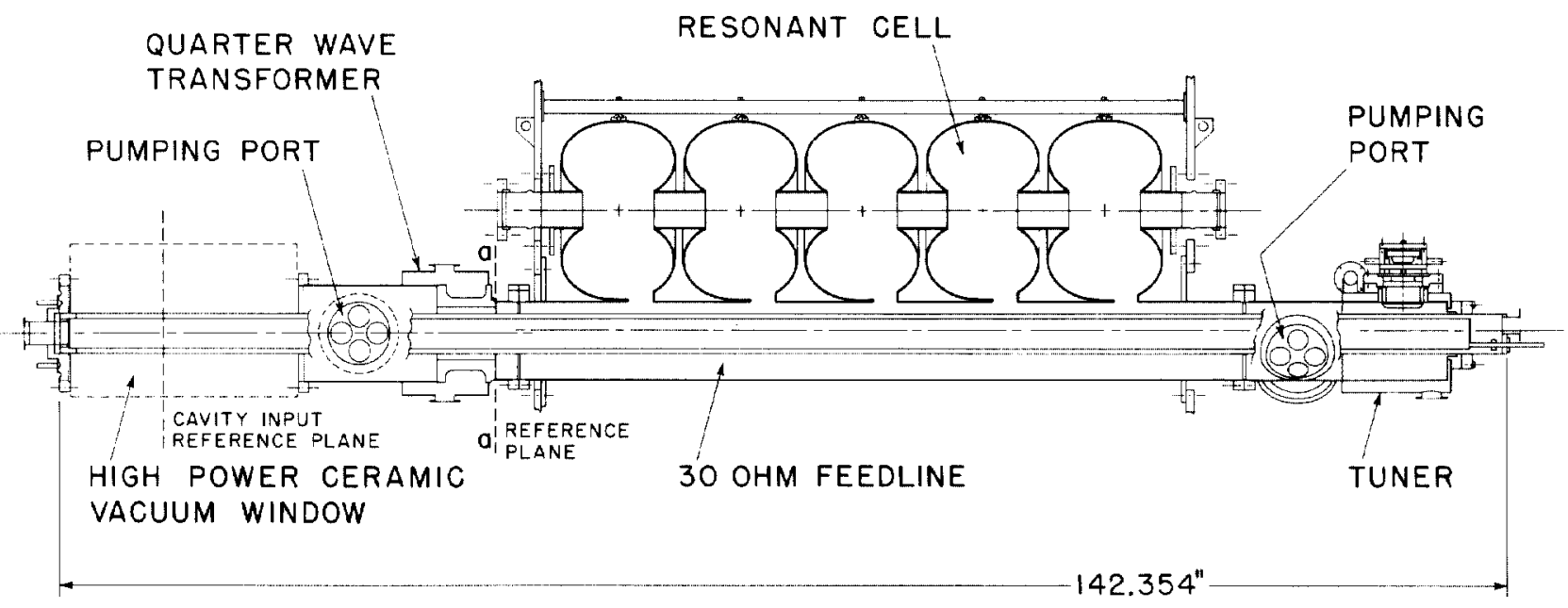

Fig. 1. The new design of CESR five cells cavity.

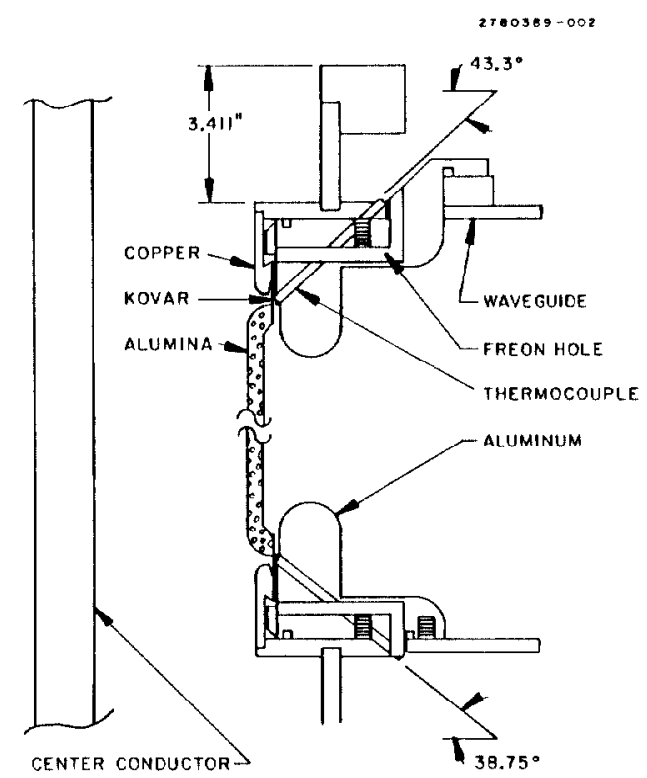

Fig. 2. High power vacuum window.

\section{Simulation}

To maximize the power transferred to the new design 5 cells cavity at $600 \mathrm{~mA}$, the RF feed line and the transformer has to be changed. In order to find these dimensions the cavity was simulated, including the strong effect of thc bcam loading on the impedances. Each cell was modelled as a parallel RLC circuit with a series inductance representing the coupling hole inductance [1]. The dependence of the shunt impedance, and its angle, without beam, at the input of the transformer (ref. plane a-a) on the tuner position is shown in Fig. 3 . This impedance is calculated using the program Touchstone [4]. Note that the resistive part is not constant as the tuner changes. This is due to the fact that the power to the cells is coupled through the coupling iris to the coax line and not through the beam hole.
Table No. 1:

\section{Design Operating Parameters}

No. of Bunches/Beam

Total Beam Current

$\sigma_{z}$

$\beta^{*}$

$Q_{x}$

$Q_{y}$

$\alpha_{p}$

$\phi_{\mathrm{s}}$

Operating Frequency

Input Power/cavity

Voltage/Cavity
No. of Cavities
14

$600 \mathrm{~mA}$

$1.7 \mathrm{~cm}$

$1.5 \mathrm{~cm}$

13.4

9.36

0.007306

69.29

4

$499.767 \mathrm{MHz}$

$300 \mathrm{~kW}$

$1.2 \mathrm{MeV}$
To minimize reflection at the cavity input the tuner position is adjusted for each current to make the impedance at reference plane a-a real [3]. Using the data shown in Fig. 3 the effective tuner length was calculated numerically for each current shown in Fig. 4. This is then used to find the power at reference plane a-a and the cavity input as shown in Fig. 5. Having standing waves in the RF feed line makes it possible for the power at reference a-a to be higher than the input power. The calculated reflection coefficient at these planes are shown in Fig. 6.

The transformer dimensions were designed to have no reflections at $600 \mathrm{~mA}$. In order to do so the beam loading resistance is first calculated from the reflection at plane a-a which is shown in Fig. 7. The power transferred to the cavity is then maximized for the design current of $600 \mathrm{~mA}$. The outer diameter of the quarter wave length transformer was designed to match the the resistance at ref. plane a-a for this' current to the $50 \mathrm{ohm}$ input line. 


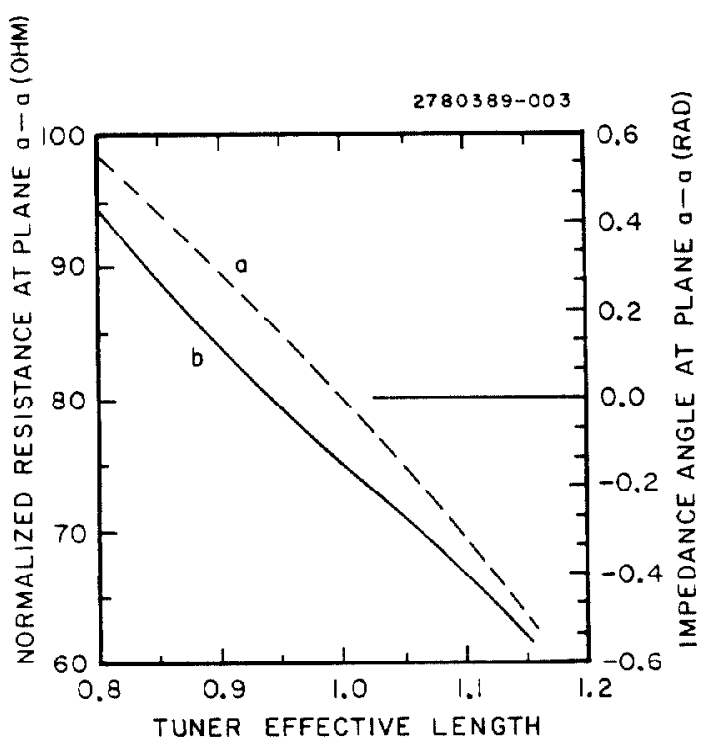

Fig. 3. Normalized shunt resistance, and impedance angle as function of the tuner effective length, at reference plane a-a.

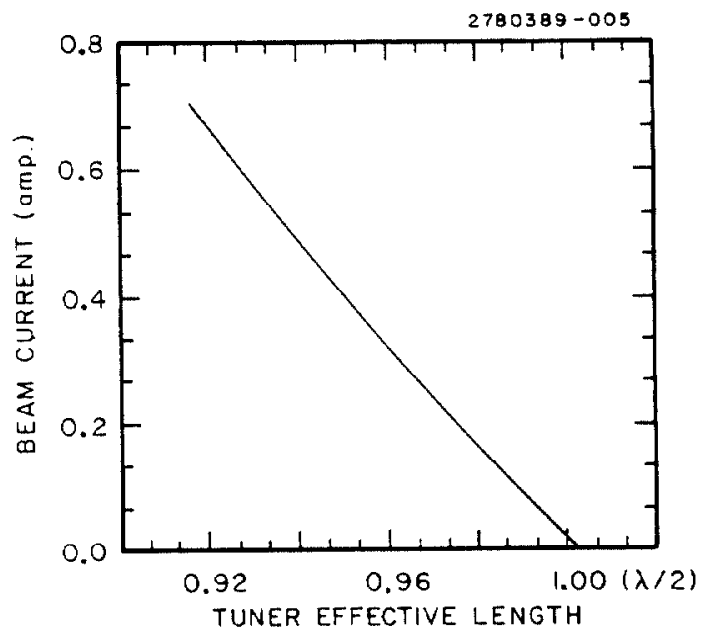

Fig. 4. The tuner effective length needed for each current for minimum reflections at cavity input.

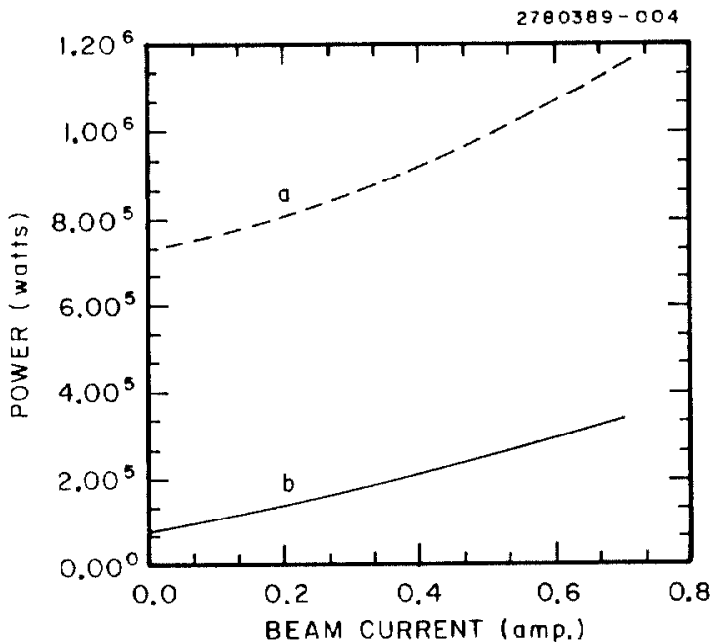

Fig. 5. The power at: a) reference plane $\mathbf{a}-\mathbf{a}, \mathbf{b}$ ) cavity input, as function of beam current.

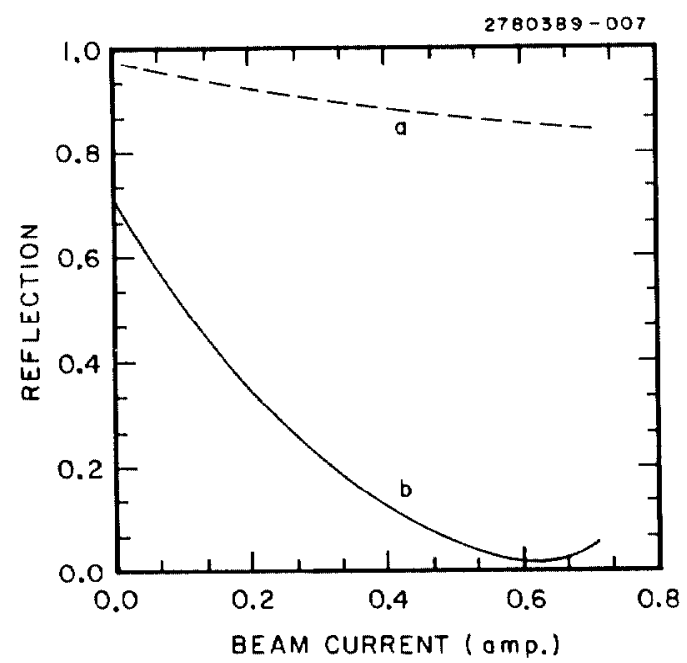

Fig. 6. The reflection at: a) reference plane a-a, b) cavity input, as function of beam current.

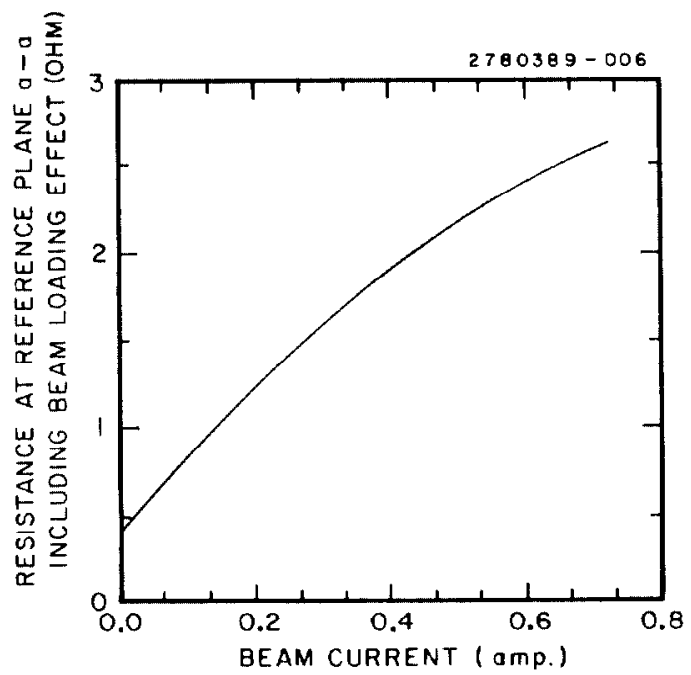

Fig. 7. Resistance at reference plane a-a including beam loading effect as function of beam current.

\section{References}

1. $\mathrm{R}$.M Sundelin, J. L. Kirchgessner, and M. Tigner, "Parallel Coupled Cavity Structure," IEEE Trans. on Nucl. SCi. NS-24, No.3, 1686 (1977).

2. R. Sundelin, M. Billing, R. Kaplan, J. Kirchgessner, R. Meller, "CESR RF System," IEEE Trans. on Nucl. Sci. NS-28, No.3, 2844 (1981).

3. P. B. Wilson, "High Energy Electron Linacs: Application to storage Ring RF Systems and Linear Colliders," SLAC-PUB -2884 (1982).

4. EEsof Inc. 